\title{
EDITORIAL
}

\section{Exercise training for pulmonary hypertension: another prescription to write?}

\author{
Lewis J. Rubin
}

F or over 30 years I have been advising my patients with pulmonary hypertension $(\mathrm{PH})$ to be physically active to a level of exertion that does not produce severe dyspnoea persisting post-exercise, dizziness, syncope or chest pain, based on the assumption that inactivity was bad both physically and mentally. This empirical advice meant little in the years before effective medical and surgical methods of treating $\mathrm{PH}$ were developed, but gained importance both as a conditioning practice for patients considered for transplantation or pulmonary endarterectomy, and as an adjunct to long-term medical therapy [1]. Only recently, however, has evidence supporting a meaningful benefit of physical activity been generated, dispelling the notion that there may be more harm than good resulting from attempting to increase blood flow through a restricted and presumed noncompliant pulmonary vascular bed [2]. In this issue of the European Respiratory Journal, GRÜNIG et al. [3] bring our understanding of the effects of exercise in $\mathrm{PH}$ a leap forward by demonstrating that an intensive 3-week in-hospital rehabilitation programme followed by a regimented home exercise programme resulted in marked improvements in a variety of exercise parameters, as well as indices of quality of life. Furthermore, these effects persisted in the cohort re-evaluated after 15 weeks of training. These results are even more impressive when one considers that similar results were seen irrespective of the aetiology and functional severity of $\mathrm{PH}$. The authors emphasise, however, that supervision and monitoring are important, since episodes of presyncope and syncope were observed, although no fatalities resulted. Taken together, these data provide guidance for instructing patients on the potential benefits and risks of intensive training.

Not all of the individual results of this study are as compelling as the sum of its parts, however. The improvements in 6-min walk test reported by GRÜNIG et al. [3] are greater than those observed in any clinical trial with medical therapy for $\mathrm{PH}$, and even more dramatic when considering that most patients were already on combination therapy. However, the 6-min walk test is highly subject to a "learning effect", even without a rigorous training regimen, and the 6-min walk results in unblinded trials have typically been much greater than those achieved in subsequent double-blind trials [4]. Furthermore, increases of $50 \mathrm{~m}$ or more have been observed in placebo-treated subjects in $\mathrm{PH}$ clinical trials [5]. In addition, reliably estimating resting

CORRESPONDENCE: L.J. Rubin, School of Medicine, University of California, San Diego, 9300 Campus Point Dr, M/C 7372, La Jolla, CA 92037, USA. E-mail: ljrubin@ucsd.edu pulmonary artery systolic pressure using Doppler echocardiography is dubious, at best, in patients with $\mathrm{PH}$ [6]; reliably estimating pressure during exercise should be considered more art than science at present. Similarly, assessing functional class can be quite subjective and susceptible to unblinding bias. Nevertheless, improvement in the more objective parameters, including maximal oxygen consumption, resting and maximal heart rate, are convincing and support benefit. That even those patients who failed to improve exercise capacity nevertheless improved their quality of life indices is strong evidence in support of a programme that incorporates physiotherapy and psychosocial support for $\mathrm{PH}$, along with medical care.

GRÜNIG et al. [3] point out that this was not a randomised, blinded trial, which would be impossible to achieve with this therapy. It is also worth noting that this is a single centre experience, and duplication of these results from other centres is needed, not only to confirm them, but to demonstrate that this aggressive programme is feasible elsewhere as well, and therefore of potential relevance to many more patients. The expense and need for the 3-week in-hospital phase should also be reconsidered, since this is not feasible in many parts of the world. Additionally, the authors applied the "last observation carried forward (LOCF)" statistical method to analyse the results of patients who did not complete the full 15-week programme. However, the non-completers comprised a large percentage $(40 \%)$ of the total population, and LOCF would give an overestimate of the "true" treatment effect if those who dropped out did so because of worsening for any cause [3]. Finally, as with medical therapy for $\mathrm{PH}$, it will be important to evaluate the maintenance and durability of these effects over a longer period of observation.

In this Olympic year we are enthralled by the gracefulness of trained athletes and reminded of the benefits of physical activity even for those of us who do not compete. GRÜNIG et al. [3] now provide evidence that one of the prescriptions that we write for our $\mathrm{PH}$ patients should be for physical activity and exercise. I can now look forward to the day when we will hold a PH Special Olympics.

\section{STATEMENT OF INTEREST}

None declared.

\section{REFERENCES}

1 Langer D, Cebrià i Iranzo MA, Burtin C, et al. Determinants of physical activity in daily life in candidates for lung transplantation. Respir Med 2012; 106: 747-754. 
2 de Man FS, Handoko ML, Groepenhoff H, et al. Effects of exercise training in patients with idiopathic pulmonary artery hypertension. Eur Respir J 2009; 34: 669-675.

3 Grünig E, Lichtblau M, Ehlken N, et al. Safety and efficacy of exercise training in various forms of pulmonary hypertension. Eur Respir J 2012; 40: 84-92.

4 Galiè N, Manes A, Negro L, et al. A meta-analysis of randomized controlled trials in pulmonary arterial hypertension. Eur Heart $J$ 2009; 30: 394-403.
5 McLaughlin VV, Benza RL, Rubin LJ, et al. Addition of inhaled treprostinil to oral therapy for pulmonary arterial hypertension: a randomized controlled clinical trial. J Am Coll Cardiol 2010; 55: 1915-1922.

6 McLaughlin VV, Archer ST, Badesch DB, et al. ACCF/AHA 2009 Expert Consensus Document on Pulmonary Hypertension. A report of the American College of Cardiology Foundation Task Force on Expert Consensus Documents and the American Heart Association. J Am Coll Cardiol 2009; 53: 1573-1619. 